\title{
Neuropil Pattern Formation and Regulation of Cell Adhesion Molecules in Drosophila Optic Lobe Development Depend on Synaptobrevin
}

\author{
Peter Robin Hiesinger, Christian Reiter, Harald Schau, and Karl-Friedrich Fischbach \\ University of Freiburg, Institute of Biology III, D-79104 Freiburg, Germany
}

To investigate a possible involvement of synaptic machinery in Drosophila visual system development, we studied the effects of a loss of function of neuronal synaptobrevin, a protein required for synaptic vesicle release. Expression of tetanus toxin light chain (which cleaves neuronal synaptobrevin) and genetic mosaics were used to analyze neuropil pattern formation and levels of selected neural adhesion molecules in the optic lobe. We show that targeted toxin expression in the developing optic lobe results in disturbances of the columnar organization of visual neuropils and of photoreceptor terminal morphology. IrreC-rst immunoreactivity in neuropils is increased after widespread expression of toxin. In photoreceptors, targeted toxin expression results in increased Fasciclin II and chaoptin but not
IrreC-rst immunoreactivity. Axonal pathfinding and programmed cell death are not affected. In genetic mosaics, patches of photoreceptors that lack neuronal synaptobrevin exhibit the same phenotypes observed after photoreceptorspecific toxin expression. Our results demonstrate the requirement of neuronal synaptobrevin for regulation of cell adhesion molecules and development of the fine structure of the optic lobe. A possible causal link to fine-tuning processes that may include synaptic plasticity in the development of the Drosophila CNS is discussed.

Key words: Drosophila; optic lobe development; synaptobrevin; synaptic plasticity; cell adhesion molecules; tetanus toxin; Fasciclin II; IrreC-rst; chaoptin
The optic lobes of Drosophila melanogaster develop a highly organized neuropil structure during pupation (for review, see Meinertzhagen and Hanson, 1993) in which $~ 120,000$ neurons form synaptic contacts. Three main steps of neuronal development-axonal pathfinding, target selection, and address selection - have been distinguished by Goodman and Shatz (1993). In the optic lobe of Drosophila, axonal pathfinding is completed within the first $20 \%$ of pupal development $(P+20 \%)$. Little is known about the following establishment of a precise pattern of synaptic connectivity.

Although the time of the first occurrence of functional synapses in the developing Drosophila optic lobe neuropils is unknown, some evidence has accumulated from various studies in different fly species. In Musca, synapses can be observed in the lamina after $\sim \mathrm{P}+55 \%$ with electron microscopy (Fröhlich and Meinertzhagen, 1982). Electrophysiological measurement reveals an onset of light-evoked photoreceptor activity in Drosophila at P $+82 \%$ (Hardie et al., 1993). Molecules that are specifically necessary for synaptic function appear to be expressed earlier. For example, synaptotagmin, a protein of the synaptic vesicle

\footnotetext{
Received April 30, 1999; revised June 14, 1999; accepted June 16, 1999.

This work was supported by Bundesministerium für Bildung und Forschung Grant 0310959 and the Deutsche Forschungsgemeinschaft. We thank S. Benzer, S. DiNardo, C. S. Goodman, K. Ito, H. Niemann, C. O'Kane, T. L. Schwarz, S. Sweeney, G. M. Technau, and the Bloomington Stock Center for supplying fly strains and reagents used in this study. We are grateful to C. M. Schuster, R. Cassada, and I. A. Meinertzhagen for helpful discussion. We also thank G. Konermann and A. Offinger for patient help with x-ray treatment, L. Falla-Christ, M. Böhler, and S. Vonderstrass for the maintenance of reagents and fly stocks, J. Köhler for the TUNEL staining protocol, and all members of the Fischbach laboratory for discussion.

Correspondence should be addressed to Dr. Karl-Friedrich Fischbach, Institute for Biology III, University of Freiburg, Schaenzlestrasse 1, D-79104 Freiburg, Germany. E-mail: kff@uni-freiburg.de

Copyright (C) 1999 Society for Neuroscience 0270-6474/99/197548-09\$05.00/0
}

cycle, can be detected immunhistochemically as early as P $+20 \%$ (X. Y. Sun and I. A. Meinertzhagen, personal communication).

Neuronal synaptobrevin (also named VAMP, abbreviated $\mathrm{n}$-syb throughout this paper), is another synapse-specific molecule that was characterized as a synaptic vesicle protein (DiAntonio et al., 1993; for review of synaptic vesicle cycle, see Südhoff, 1995). The analysis of n-syb in Drosophila revealed its function downstream of neurotransmitter vesicle docking (Broadie et al., 1995; for review, see Wu and Bellen, 1997). The analysis was based on the targeted expression of tetanus toxin light chain (TeTxLC) via the Gal4/UAS ectopic expression system (Brand and Dormand, 1995). TeTxLC specifically eliminates evoked synaptic transmission by selectively cleaving n-syb in Drosophila (Sweeney et al., 1995). Transformants expressing TeTxLC throughout the embryonic nervous system and null mutants of n-syb (Deitcher et al., 1998) both die at the end of embryogenesis.

Neither the onset and consequences of $n$-syb expression in the developing pupal CNS nor any involvement of synaptic machinery in Drosophila CNS development have been elucidated until now.

An involvement of cell adhesion molecules (CAMs) in synaptic plasticity was first demonstrated by Mayford et al. (1992) in Aplysia by showing that apCAM is downregulated in sensory neurons after application of the neurotransmitter serotonin. Downregulation of Fasciclin II (FasII), the Drosophila homolog of apCAM and NCAM, is necessary and sufficient for presynaptic sprouting of motoneurons in Drosophila (Schuster et al., 1996a,b). A dependence of Drosophila development on neuronal activity was first demonstrated by Broadie and Bate (1993) for the neuromuscular junction.

In this paper we report the requirement of neuronal synaptobrevin for neuropil pattern formation as well as for the regulation of the CAMs Fasciclin II, IrreC-rst, and chaoptin in the Drosoph- 
ila optic lobe. We used targeted TeTxLC expression to inactivate $\mathrm{n}$-syb in a variety of optic lobe neurons and specifically in photoreceptors. Effects similar to those observed after TeTxLC expression were seen in genetic mosaics for n-syb in photoreceptors.

\section{MATERIALS AND METHODS}

Fly strains, Gal4 screen, and conditions of culture. For Gal4/UAS crosses the following lines were used: Gal4 lines: Mz1369 (obtained from G. Technau, University of Mainz, Mainz, Germany) and GMR-Gal4 (Freeman, 1996; obtained from Bloomington Stock Center); UAS lines: UASGFP-S65T (obtained from K. Ito, Okazaki, Japan) and TeTxLC; and UAS-TNT lines (insertions of active TeTxLC: UAS-TNT-H, UASTNT-C, and UAS-TNT-E; insertions of inactive TeTxLC: UAS-TNT-V and UAS-TNT-Q) from S. Sweeney (University of Cambridge, Cambridge, UK) (Sweeney et al., 1995). In the first screen, the offspring of crosses of 20 selected Gal4 lines with the UAS lines containing TeTxLC constructs were tested for their ability to survive until adulthood. The expression pattern of selected Gal4 lines was then analyzed using the green fluorescent protein (GFP) as a reporter for preferential expression in the optic lobe during pupal development. For fly strains used in mosaic experiments, see below. N-syb hypomorph mutants were produced by crossing $\mathrm{n}$-syb null flies $\Delta \mathrm{F} 33 \mathrm{~B}$ with either line I4 or I18 (ethyl methyl sulfonate-induced n-syb hypomorph; all lines described by Deitcher et al., 1998). WTB was used as wild-type stock. Flies were raised at $25^{\circ} \mathrm{C}(100 \%$ pupal development corresponding to $103 \mathrm{hr}$ ).

Immunohistochemistry. Larval, pupal and adult brains were prepared in Ringer's solution and immediately fixed in $4 \%$ paraformaldehyde (15-45 $\mathrm{min})$. The brains were subsequently put into 1\% NGS blocking solution for $30 \mathrm{~min}$ and incubated with primary antibody for $6-12 \mathrm{hr}$ at $4^{\circ} \mathrm{C}$. Dilutions for the antibodies used: 1D4, 1:100; 24A5, 1:50; nc82,1:20; 24B10, 1:200; anti-TNT, 1:20; and anti-n-syb, 1:100. We used secondary antibodies with $\mathrm{Cy} 3$ and DTAF labeling (Jackson ImmunoResearch, West Grove, PA). Washing between all steps used PBS with $0.4 \%$ Triton X-100 (PBT). Preparations were embedded in Vectashield (Vector Laboratories, Burlingame, CA). To determine differences in staining intensities, mixing experiments were performed in which brains of two genotypes were prepared simultaneously and subsequently handled in the same tube for fixation as well as all staining, washing, and mounting steps. To distinguish the two genotypes, the brains of one type were cut into halves during preparation. A second experiment with the brains of the other type cut into halves was made for symmetric comparison. Such specimens were subsequently scanned with the same settings and in the same session with the confocal microscope.

Confocal laser scanning microscopy and three-dimensional image processing. A Leica (Nussloch, Germany) TCS4D confocal microscope equipped with an ArKr laser was used for data acquisition. Series of complete optic lobes were scanned with a $40 \times$ objective (aperture 1.0) and comprised 182 images of $512 \times 512$ pixel resolution at 8 bit color depth. Such data were three-dimensionally reconstructed on an SGI (Mountain View, CA) Octane MXI workstation using the threedimensional (3D) visualization software Amira (Konrad Zuse Zentrum, Berlin, Germany) and Imaris (Bitplane, Zurich, Switzerland). Algorithms used comprise cross-talk correction, maximum projection, averaging, and blending of image stacks as well as maximum projection and ray tracing of complete or cut $3 \mathrm{D}$ volumes. High-resolution scans were made with an $100 \times$ objective (aperture 1.4). Figures were assembled and labeled in Adobe (San Jose, CA) Photoshop.

TdT-mediated biotin-dUDP nick-end labeling apoptosis staining. The method of TdT-mediated biotin-dUDP nick-end labeling (TUNEL) has been described elsewhere (Gavrieli et al., 1992). We used the in situ cell death detection POD kit from Boehringer Mannheim (Mannheim, Germany) to show apoptosis in whole mounts. Brains were prepared and fixed as described above and subsequently treated with proteinase $\mathrm{K}$ (10 $\mu \mathrm{g} / \mathrm{ml}$ ) for $5 \mathrm{~min}$ and $2 \mathrm{mg} / \mathrm{ml}$ glycine in PBT solution for $3 \mathrm{~min}$. Brains were then fixed with $4 \%$ paraformaldehyde for the second time and washed with PBT and PBS. After dehydration, endogenous peroxidase was blocked with $0.3 \% \mathrm{H}_{2} \mathrm{O}_{2}$ in methanol. After rehydration, TUNEL labeling with POD 1 and 2 solutions (enzyme and labeling solutions, respectively) was incubated for $60 \mathrm{~min}$ at $37^{\circ} \mathrm{C}$, washed with $\mathrm{PBS}$, and subsequently incubated with Converter-POD (anti-fluorescein antibody conjugated with horseradish peroxidase). The peroxidase reaction was performed with DAB solution and stopped with $0.3 \% \mathrm{H}_{2} \mathrm{O}_{2}$. The preparations were analyzed and documented with a Zeiss (Thornwood, NY) Axiophot microscope.

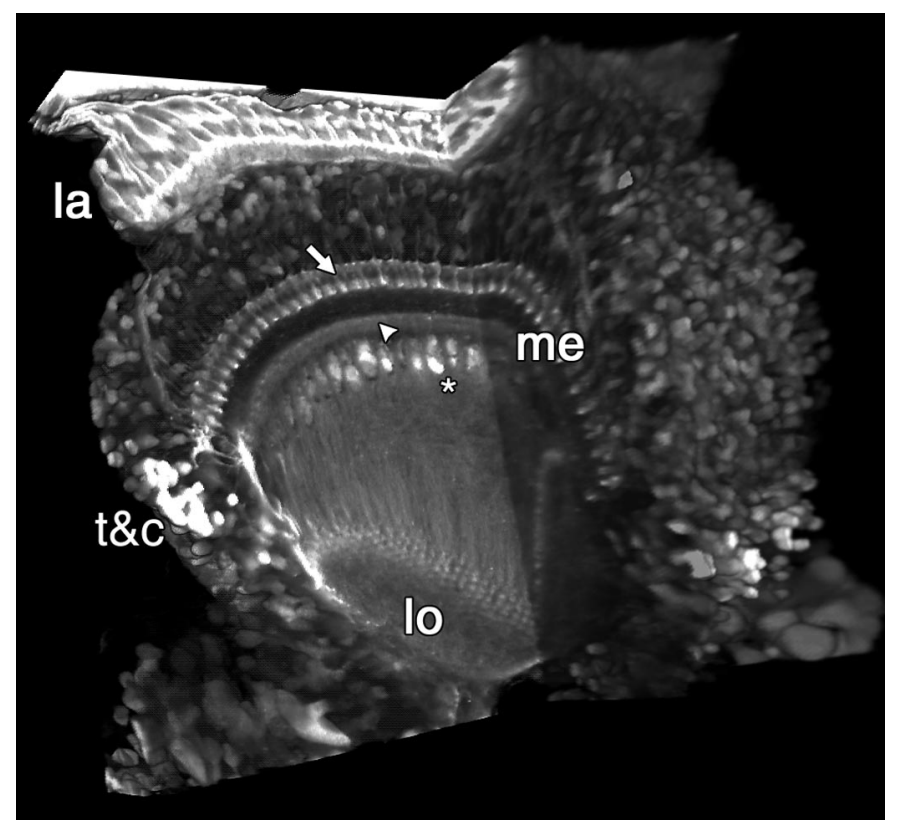

Figure 1. Cutaway 3D volume reconstruction of Mz1369-driven GFP at $\mathrm{P}+25 \%$. Mz1369 drives expression in a large variety of neurons in the developing optic lobe. GFP expression can be detected in the distal (arrow) and proximal (arrowhead) parts of the medulla (me) as well as in glial cells (asterisk) in the inner chiasm. lo, Lobula complex; $t \& c$, T- and $\mathrm{C}$-cells; la, lamina. The reconstruction represents a volume of $250 \times$ $250 \times 92 \mu \mathrm{m}$.

Genetic mosaic analysis. White-eyed n-syb mutants yw; $\Delta$ F33B/TM6 $\mathrm{y}+$ (Deitcher et al., 1998) were crossed to two lines containing P elements with the white gene as marker near the n-syb locus on the third chromosome [line 1: $\mathrm{y}^{*} \mathrm{w}^{*} ; \mathrm{P}\{\mathrm{w}+\mathrm{mC}=1 \mathrm{acW}\} 1(3) \mathrm{j} 4 \mathrm{~A} 6 \mathrm{j} 4 \mathrm{~A} 6 / \mathrm{TM} 6 \mathrm{~B}$, AntpHu $\mathrm{Tb}+$ (from Bloomington Stock Center); line 2: $\mathrm{yw} ; \mathrm{P}\{\mathrm{w}+$; lacZ[34]; 62A/B \} (from S. DiNardo, University of Pennsylvania)]. Mosaics were created by $\mathrm{x}$-ray treatment $(8-16 \mathrm{~Gy}$, corresponds to $800-1600$ $\mathrm{rad}$ ) of the heterozygotes after $41 \pm 5 \mathrm{hr}$ after egg laying at $25^{\circ} \mathrm{C}$. Adult offspring was screened for white or red eye spots. Mosaic eyes were photographed, and the individual brains were subsequently prepared, stained with monoclonal antibodies (mAbs) 1D4, 24A5, or 24B10, and mounted as described above.

\section{RESULTS}

\section{Targeted tetanus toxin light chain expression produces neuropil irregularities during optic lobe development}

To test the influence of TeTxLC expression on developing optic lobe neuropils, we screened existing Gal4 lines for high expression levels in the optic lobe during metamorphosis (see Materials and Methods). The enhancer trap Gal4 line Mz1369 fitted the selection requirements. Active TeTxLC-expressing flies (UAS-TNT-H; Sweeney et al., 1995) survive up to the end of metamorphosis, but most do not eclose, and all flies die within the first hours of adulthood (data not shown). In contrast, flies expressing point-mutated inactive TeTxLC (UAS-TNT-V) have no obvious developmental deficits. Mz1369 exhibits high levels of expression in many cell types of the optic lobe (Fig. 1) throughout pupation. Among expressing cells in different time windows are photoreceptors, lamina monopolar cells, as well as C- and T-cells projecting through the inner chiasm. Almost no expression can be seen in the developing central brain of larvae and early pupae. Central brain expression becomes stronger thereafter and is widespread in the complete brain at adulthood (data not shown).

The neuropil morphology of active and inactive toxinexpressing pupae was analyzed using two sets of monoclonal 

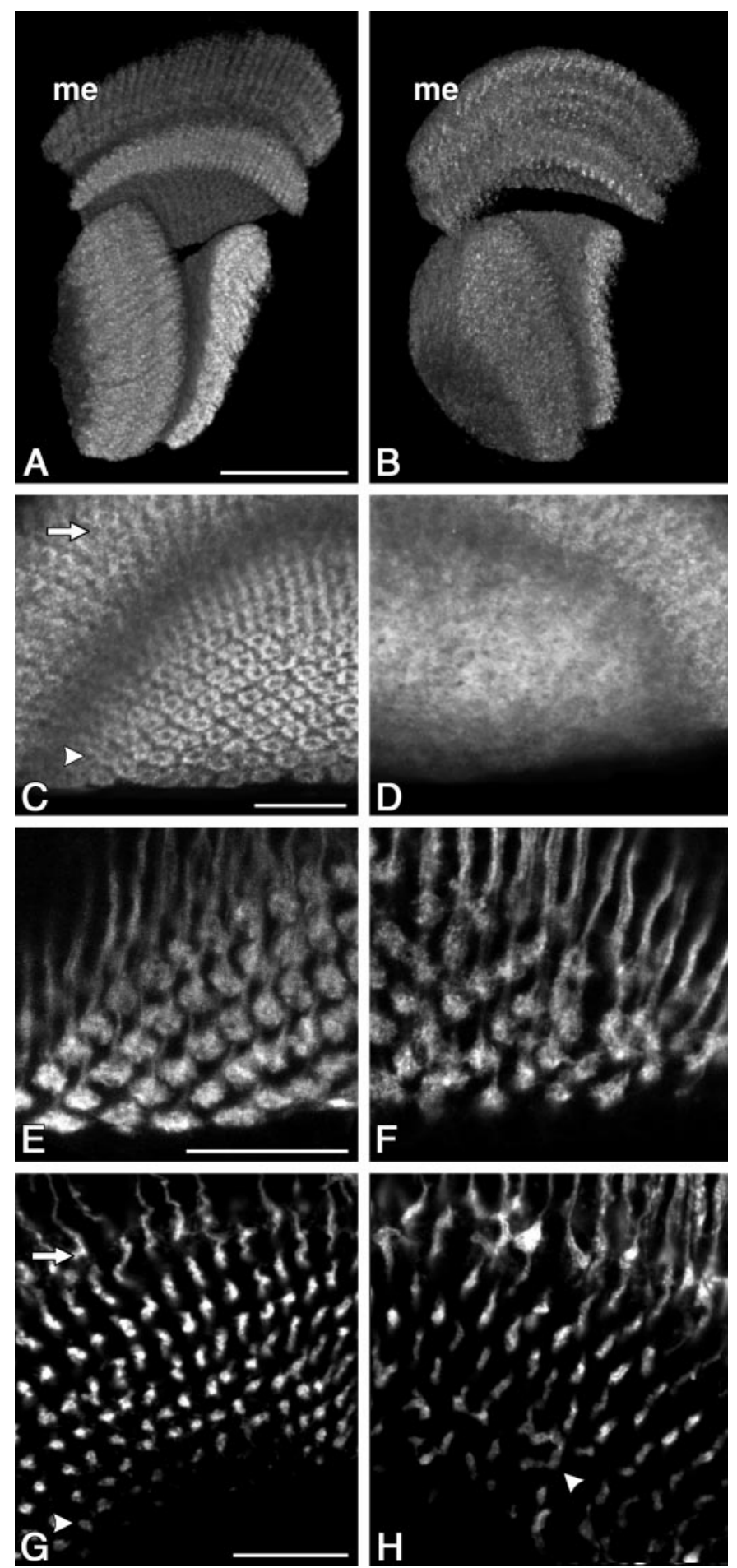

Figure 2. Widespread expression of active TeTxLC (inactive, Mz1369/ UAS-TNT-V; active, Mz1369/UAS-TNT-H) alters medulla patterning in the midpupal Drosophila optic lobe. $A$, $B$, Horizontally sectioned, partial reconstructions of $\mathrm{mAb}$ nc82-stained optic lobes. The wild-type columnar organization of the medulla $(\mathrm{me})$ is clearly recognizable after expression of inactive TeTxLC $(A)$ but appears disturbed after expression of active TeTxLC $(B)$. The overall shape of the neuropils is not altered. $C, D$, Medulla stainings of inactive and active TeTxLC-expressing optic lobes with anti-IrreC. As in wild type, expression of inactive TeTxLC produces a highly organized staining pattern of the distal (arrow) and proximal (arrowhead) medulla. A complete loss of columnar organization in the proximal medulla (9th layer) can be seen after active TeTxLC expression $(D) . E$, $F$, Staining with anti-FasII shows arborizations of Fasciclin IIpositive lamina monopolar cells in the distal medulla. The wild-type pat- antibodies: markers for pupal neuropils (mAb nc82 and antiIrreC; Fig. 2A,C) and markers for characterized neuronal cells that form arborizations in the medulla (anti-FasII and antichaoptin; Fig. 2E,G). Anti-IrreC (mAb 24A5; Schneider et al., $1995)$ is directed against the cell adhesion molecule IrreC-rst, which is expressed on visual fibers during axonal pathfinding and subsequently persists in the optic lobe neuropils. In contrast, almost the complete neuropil of Drosophila during all stages of development is stained with mAb nc82, an antibody against an unknown neuronal antigen (Laissue et al., 1999). Anti-chaoptin (mAb 24B10; Zipursky et al., 1984) is an antibody against chaoptin, which is a photoreceptor-specific CAM involved in rhabdomere formation (Van Vactor, 1988; Krantz and Zipursky, 1990). Anti-FasII (mAb 1D4) is directed against the cell adhesion molecule Fasciclin II (Lin et al., 1994), which is expressed in the optic lobes by lamina monopolar cells L1 and L3 as well as C-and T-cells. Fasciclin II immunoreactivity in the optic lobe is downregulated at the end of morphogenesis (Schneider et al., 1995). Anti-chaoptin as well as anti-FasII stain complete fibers with their terminal arborizations in the distal medulla, so we concentrated on this neuropil to investigate morphological changes of terminals after axonal pathfinding.

All antibody stainings of midpupal optic lobes with Mz1369driven active toxin expression reveal severe neuropil patterning disturbances. mAb nc82 staining reveals a loss of clear columnar separations (Fig. 2B). Correspondingly, staining of IrreC-rst positive medulla layers shows a complete loss of columnar organization in the ninth layer and disturbances in distal layers (Fig. 2D). Arborizations of L1 and L3 stained with the Fasciclin II antibody as well as arborizations of photoreceptors R7 and R8 stained with the chaoptin antibody exhibit severe morphological irregularities at this stage. Arborizations and terminals that form regular patterns in wild type and in flies expressing inactive toxin overlap irregularly when active toxin is expressed (Fig. $2 E-H$ ).

Because expression in Mz1369 is widespread and dynamic in the developing optic lobe, it cannot be inferred from these experiments whether the influence of TeTxLC is merely presynaptic and cell-autonomous or extends to postsynaptic cells. To analyze the influence of TeTxLC expression in a known cell type, we thus used the GMR-Gal4 line, which drives expression in all photoreceptor neurons throughout development under control of the glass DNA-binding site (Moses and Rubin, 1991; Freeman, 1996).

In optic lobes expressing either active or inactive toxin in photoreceptors, antibody staining with the anti-TeTxLC antibody (Sweeney et al., 1995) closely matches the staining pattern of anti-Chaoptin, including fine arborizations. In addition to antichaoptin, we therefore used anti-TeTxLC to analyze R7 and R8 axons and terminals and at the same time to confirm the restricted occurrence of TeTxLC protein.

At $\mathrm{P}+50 \%$ and later, GMR-Gal4- and Mz1369-driven active toxin produces indistinguishable defects of photoreceptor terminal morphology (compare Figs. $2 H, 3 J$ ). Correspondingly, in a

tern of arborizations is observed after inactive TeTxLC expression $(E)$. After active TeTxLC expression the arborizations appear disorganized and show overlaps $(F) . G, H$, Staining with anti-chaoptin shows R7 and R8 photoreceptor axons and terminals in the distal medulla. Arborizations are formed in the distal medulla (arrow), and R7 terminals can be addressed in the sixth medulla layer (arrowhead). These arborizations appear regular after expression of inactive TeTxLC $(G)$ and exhibit broader extensions and more overlaps (arrowhead) after expression of active TeTxLC $(H)$. Scale bars: (in $A$ ) $A, B, 50 \mu \mathrm{m}$; (in $C, E, G) C-H, 20 \mu \mathrm{m}$. 

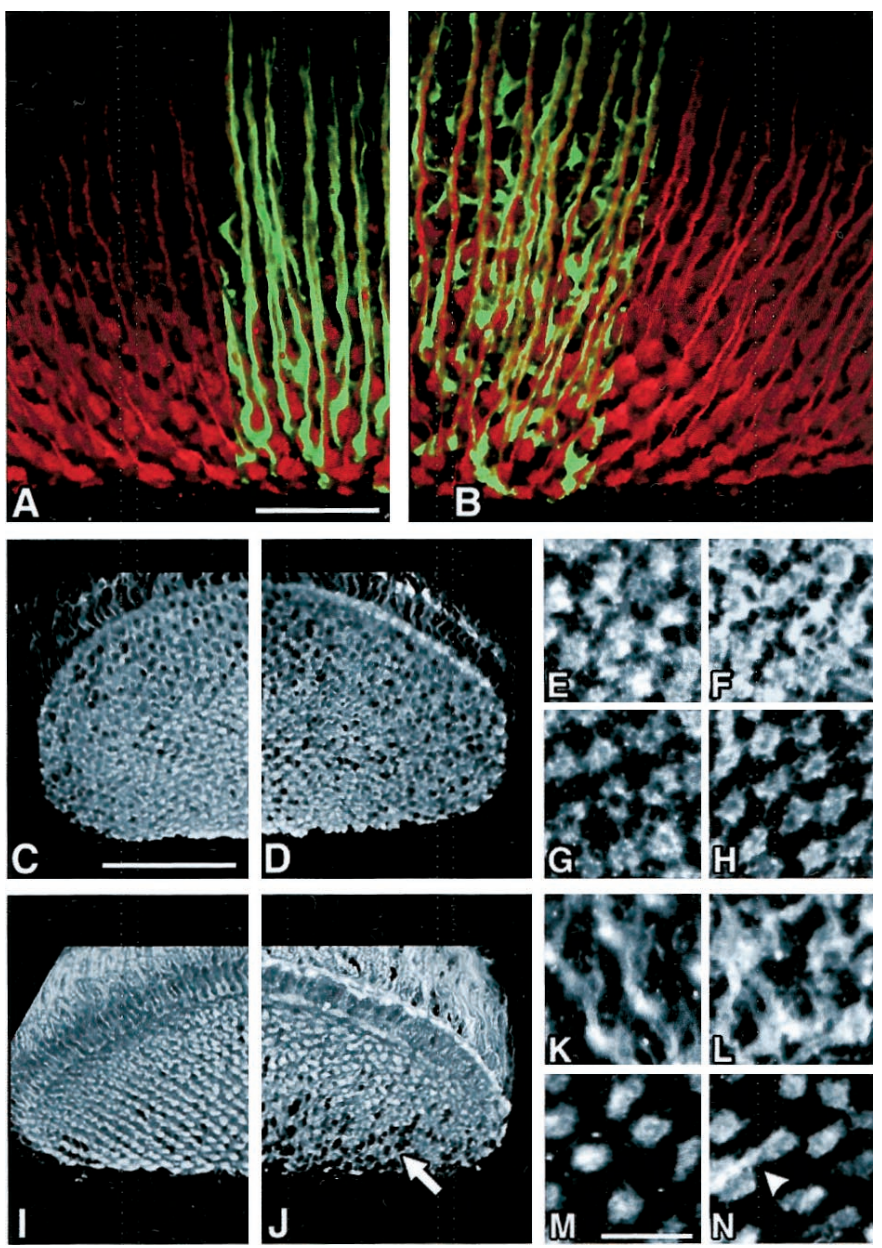

Figure 3. Photoreceptor-specific expression of active TeTxLC selectively alters photoreceptor terminal morphology. $A, B$, Reconstruction of a distal view of the medulla at $\mathrm{P}+50 \%$. As in wild type, photoreceptors (green, anti-TeTxLC staining) and lamina monopolar cells (red, antiFasII staining) form organized arborizations when entering the medulla after inactive TeTxLC expression $(A)$. Expression of active TeTxLC results in a disorganized arborization pattern of photoreceptors in the distal medulla, but the arborization pattern of lamina monopolar cells remains organized $(B)$. In the left half of $A$ as well as in the right half of $B$, photoreceptors have been removed from the reconstruction to reveal lamina monopolar cell arborizations. $C, D$, Proximal view into the terminal field of anti-chaoptin-stained photoreceptors in the medulla at $\mathrm{P}+$ 25\%. R7 and R8 photoreceptor terminals cover a large field of the medulla in inactive $(C)$ and active $(D)$ TeTxLC-expressing specimens as in wild type. $E-H$, High-resolution confocal scans show many overlaps of terminals but no significant differences between arborizations in the distal medulla under conditions of inactive $(E)$ and active $(F)$ TeTxLC expression in photoreceptors at $\mathrm{P}+25 \%$. The corresponding terminal fields in the proximal medulla remain equally unaltered by expression of active TeTxLC $(G, H) . I, J$, Proximal view into the terminal field of antichaoptin-stained photoreceptors in the medulla at P $+50 \%$. R7 terminals exhibit a characteristic bulbous morphology and form a highly organized pattern with only rare overlaps in the control $(I)$. Many overlaps, especially in the anterior part of the medulla (arrow), are evident after active TeTxLC expression ( $J$, arrowhead in $N$ ). $K-N$, High-resolution confocal scans demonstrate a significantly altered arborization morphology in the distal medulla. Fibers entering the medulla are massively interconnected with broad overlaps in the active TeTxLC background $(L)$. Correspondingly, R7 terminals in the sixth layer are often fused after active TeTxLC expression $(N)$ in comparison with the organized terminal pattern of the control $(M)$. Scale bars: (in $A$ ) $A, B, 20 \mu \mathrm{m}$; (in $C$ ) $C, D, I, J, 50 \mu \mathrm{m}$; (in $M$ ) $E-H, K-N, 10 \mu \mathrm{m}$. double labeling of midpupal GMR-Gal4/UAS-TNT-H optic lobes using the antibodies against Fasciclin II and TeTxLC, the $\mathrm{mAb}$ anti-TeTxLC-stained photoreceptors exhibit disorganized arborizations (Fig. $3 A, B$ ). Thus, cell-specific TeTxLC expression is sufficient to produce the phenotype in photoreceptors. In contrast, the Fasciclin II-positive L1 and L3 cells, which are postsynaptic to photoreceptors R1-R6 (Meinertzhagen and O'Neil, 1991), develop arborizations in the distal medulla that appear normal at the level of confocal microscopy (Fig. 3A,B). These results indicate that the effect of TeTxLC is cell-autonomous.

To investigate the onset of TeTxLC induced morphological disturbances, we studied earlier stages of pupal development. It has previously been demonstrated that early terminal arborizations of R7 and R8 broadly overlap in the developing medulla. Overlapping filopodia are extensively reduced during the first half of pupation (Ashley and Katz, 1994). At P $+25 \%$ inactive and active TeTxLC-expressing photoreceptors are indistinguishable at the level of confocal microscopy (Fig. $3 \mathrm{C}-\mathrm{H}$ ). Between $\mathrm{P}$ $+25 \%$ and $\mathrm{P}+50 \%$, a significant alteration of terminal morphology takes place that is characterized by a reduction of overlapping filopodia (Fig. 3C,I). It is in this midpupal phase of fine tuning that the TeTxLC-induced phenotype has its origin (Fig. $3 J)$. Hence, targeted TeTxLC expression produces effects in R7 and $\mathrm{R} 8$ terminals in a time window after axonal pathfinding and target selection.

\section{Targeted TeTxLC expression alters levels of cell adhesion molecules}

Because of the known essential role of Fasciclin II in synaptic plasticity and synapse formation (Schuster et al., 1996a,b; Davis et al., 1997), we asked whether TeTxLC expression and the resulting block of putative neuronal activity during pupation would affect the expression dynamics of Fasciclin II. Another CAM of the Ig superfamily, IrreC-rst, was chosen as a candidate for a synaptic adhesion molecule because of its restriction to optic lobe neuropils after $\mathrm{P}+25 \%$ (Schneider et al., 1995). Because alterations were found in the immunoreactivity of Fasciclin II and IrreC-rst, chaoptin, a photoreceptor-specific CAM of a different class (the leucine-rich repeat family) was additionally analyzed for cell type-specific alteration of immunoreactivity. No role of chaoptin in axonal pathfinding, target recognition, or synaptic development has been described so far. All three CAMs were previously shown to mediate homophilic adhesion in vitro (Krantz and Zipursky, 1990; Grenningloh et al., 1991; Schneider et al., 1995) but exert different functions in vivo.

\section{Widespread TeTxLC expression}

Anti-IrreC stainings of late pupae and adults with active toxin expression driven by Mz1369 exhibit much higher levels of IrreCrst immunoreactivity in optic lobe neuropils than the corresponding inactive toxin controls (Fig. 4A,B) and wild type, in which IrreC-rst is almost completely downregulated after $\mathrm{P}+75 \%$ (Schneider et al., 1995). In Fasciclin II antibody stainings an increased immunoreactivity was mainly detected on fibers in the inner chiasm (data not shown).

\section{Photoreceptor-specific TeTxLC expression}

In optic lobes of flies with GMR-Gal4-driven active toxin expression, IrreC-rst immunoreactivity is downregulated normally after the midpupal stage, and adult optic lobes exhibit no difference from wild type at the level of confocal microscopy (data not 

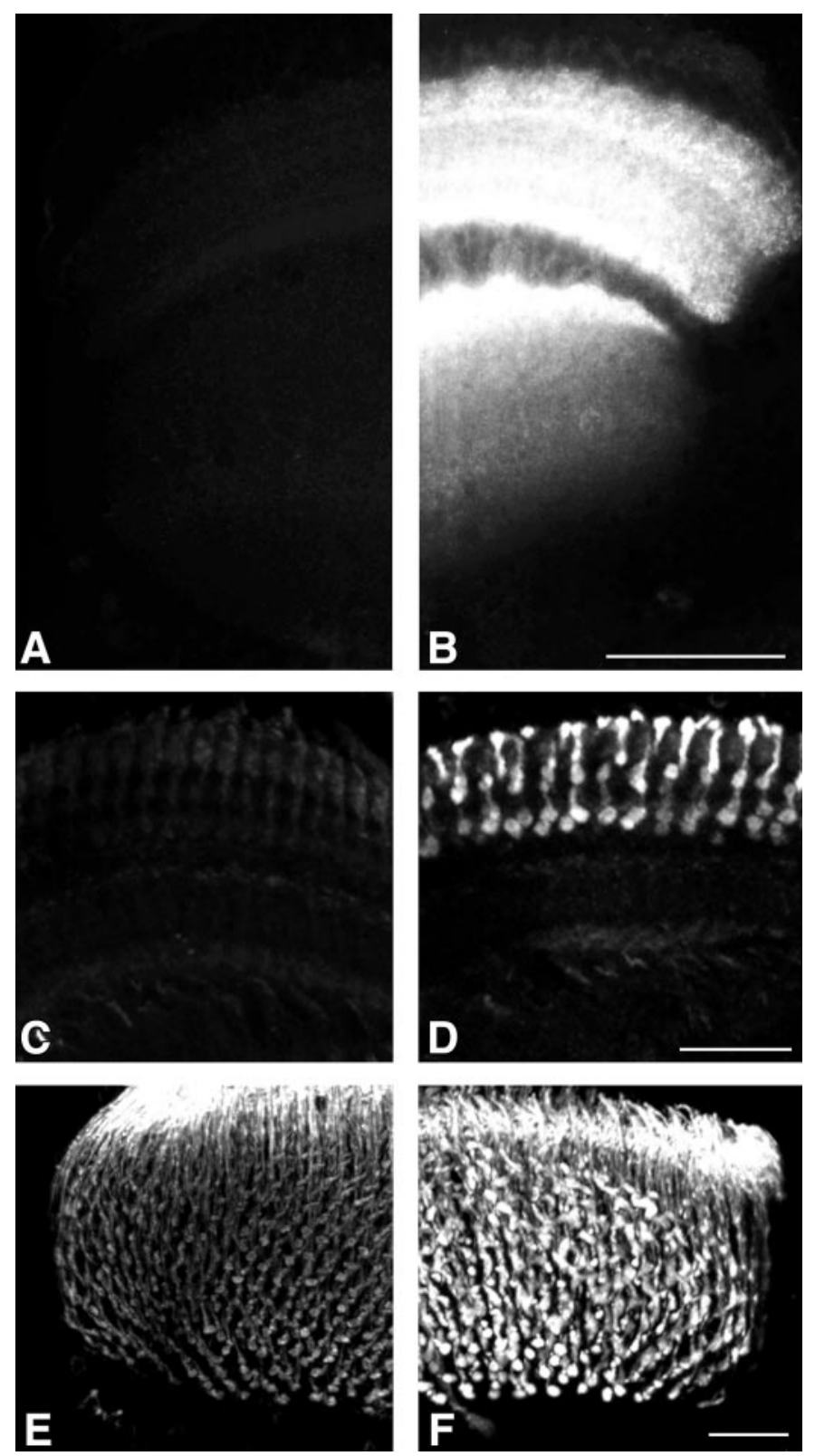

Figure 4. TeTxLC-induced alteration of IrreC-rst, Fasciclin II, and chaoptin levels. $A, B$, IrreC-rst stainings of late pupal optic lobes $(\mathrm{P}>$ $90 \%$ ). Widespread expression of inactive $(A)$ and active $(B)$ TeTxLC under control of Mz1369 results in high immunoreactivity in anti-IrreC stainings only after active TeTxLC expression. The brains were simultaneously prepared, stained in the same tube, and scanned with equal settings with the confocal microscope (see Materials and Methods). C, D, Anti-FasII stainings of adult optic lobes (maximum projections of 10 confocal images). Photoreceptor-specific expression of active (D) TeTxLC under control of GMR-Gal4 produces intense staining in R7 and $\mathrm{R} 8$ axons and terminals in the distal medulla that is missing with inactive TeTxLC $(C)$. The specimens were selected for smooth eyes. $E, F$, Antichaoptin stainings of adult optic lobes (maximum projections of 40 confocal images covering the complete depth of photoreceptor projections in the distal medulla). Again, GMR-Gal4 was used to drive photoreceptor-specific inactive and active TeTxLC expression. The immunoreactivity is significantly weaker in the control $(E)$ than after active TeTxLC expression $(F)$. The specimens were also selected for smooth eyes and were equally prepared, stained, and scanned (see Materials and Methods). Scale bars: (in $B$ ) $A, B, 50 \mu \mathrm{m}$; (in $D, F$ ) $C, D, E, F, 20 \mu \mathrm{m}$. shown). Although IrreC-rst is expressed in photoreceptors during pupation, as revealed by strong immunoreactivity in rhabdomeres (C. Reiter, unpublished results), its regulation and localization in these cells are apparently independent of n-syb function. In contrast, high Fasciclin II immunoreactivity can be shown on R7 and R8 axons and terminals in such optic lobes (Fig. $4 D$ ). In corresponding inactive controls as well as in wild-type adult optic lobes only very weak Fasciclin II immunoreactivity is detected, whereas mushroom body immunoreactivity of Fasciclin II remains at a high level, serving as an internal staining control. To answer the question of whether the high levels of Fasciclin II on photoreceptors are a result of late upregulation or rather of failed downregulation, we analyzed anti-FasII stainings of optic lobes from wild type and animals expressing active or inactive toxin in photoreceptors. No evident Fasciclin II localization at R7 or R8 terminals can be seen in any of these in midpupal stages. However, in GMR-Gal4/UAS-TNT-H optic lobes Fasciclin II staining of R7 and R8 terminals can be clearly detected from P + $75 \%$ onward (data not shown), showing an upregulation of Fasciclin II immunoreactivity in TeTxLC-expressing photoreceptors in the last quarter of pupation. To compare the chaoptin immunoreactivity of GMR-Gal4/UAS-TNT-V and GMR-Gal4/UASTNT-H optic lobes, a staining protocol was used that ensured identical treatment throughout preparation, staining, and mounting procedures (see Materials and Methods). R7 and R8 axons and terminals in adults with GMR-Gal4-driven active toxin exhibit a significantly higher immunoreactivity than in the inactive toxin-expressing controls (Fig. 4E,F). Investigation of pupal stages revealed smaller differences of chaoptin staining intensities. Nevertheless, slight differences can be observed as early as $\mathrm{P}$ $+25 \%$ (data not shown).

\section{TeTxLC expression has no influence on apoptosis or axonal pathfinding}

Although earlier work on TeTxLC does not suggest any other capability than the n-syb cleaving function described (Sweeney et al., 1995), alternative functions that might produce the phenotypes observed in this paper have to be considered. To check for an influence of high levels of toxin expression on cell survival, optic lobes expressing active or inactive TeTxLC were analyzed for irregularities in the number of cells undergoing apoptosis at $\mathrm{P}$ +25 and $\mathrm{P}+50 \%$ using the TUNEL staining technique (Gavrieli et al., 1992). Again, Mz1369 was used to drive active and inactive toxin expression in many cells (Fig. 1). No obvious difference can be found at $\mathrm{P}+25 \%$, when large numbers of cells in the optic lobe cortices undergo apoptosis regularly. At $\mathrm{P}+$ $50 \%$ apoptosis almost vanishes in wild type (Fischbach and Technau, 1984) as well as under conditions of TeTxLC expression (data not shown). These results show that high levels of active TeTxLC expression have no influence on cell survival in a large set of optic lobe cells. Furthermore, we did not observe misprojections of any TeTxLC-expressing axon fibers, supporting the view that TeTxLC actually functions at the level of terminal development but not axonal growth.

GMR-Gal4 flies have a severe eye roughness phenotype on their own, and crosses with UAS-TNT-H more often result in rough-eyed F1 flies than crosses with UAS-TNT-V. We found that approximately one-third of adult GMR-Gal4/UAS-TNT-H flies exhibit patterning defects in the eye. We therefore analyzed to what extent the morphological and CAM regulation phenotypes we observed might be a consequence of disturbed eye patterning. Rough- and smooth-eyed specimens from such crosses 

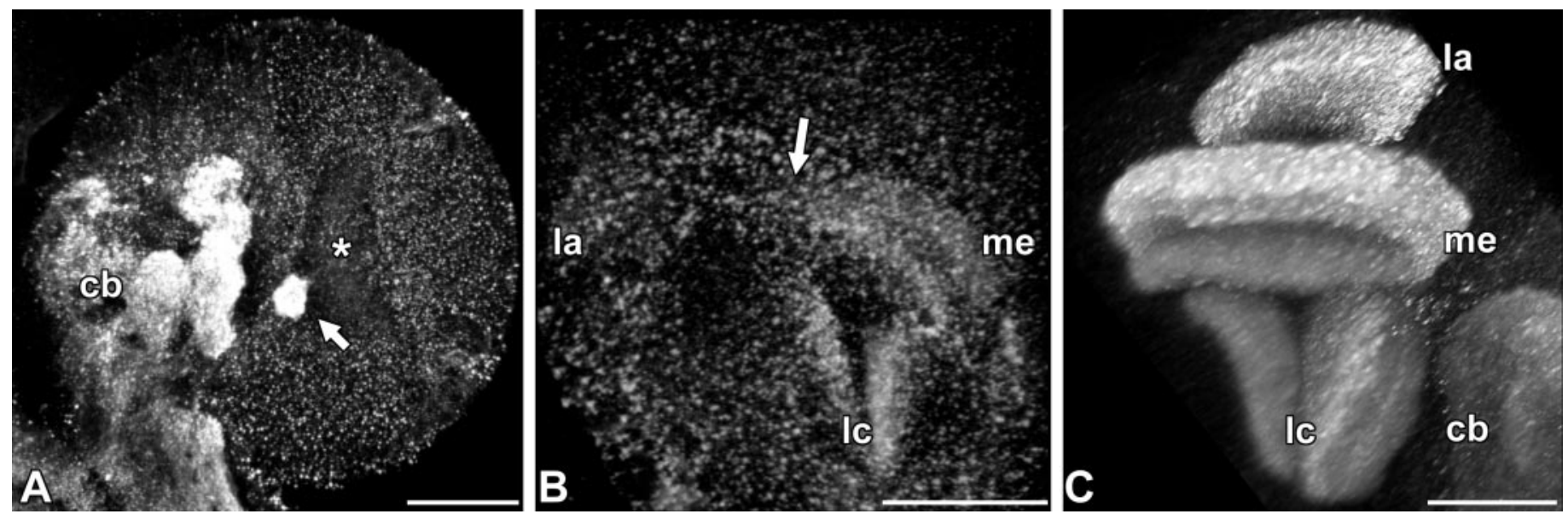

Figure 5. Developmental dynamics of synaptobrevin immunoreactivity. Larval, early, and late pupal brains were stained with n-syb antiserum (Deitcher et al., 1998). $A$, Third instar larval hemisphere. Strong staining can be detected in active neuropils: central brain (cb) and the larval optic neuropil (arrow). In contrast, the developing optic lobe (asterisk) remains almost unstained. B, Optic lobe at $\mathrm{P}+15 \%$. The neuropils of the developing optic lobe (la, lamina; me, medulla; $l c$, lobula complex) already exhibit weak synaptobrevin immunoreactivity. In this horizontal section, a gradient of decreasing immunoreactivity can be seen toward the younger part of the medulla (arrow). C, Late pupal optic lobe (P > 90\%). All neuropils exhibit high levels of synaptobrevin. Scale bars, $50 \mu \mathrm{m}$.

were analyzed for differences in stainings with the antibodies against chaoptin, Fasciclin II, and IrreC-rst. All phenotypes described in this paper were found to be indistinguishable. Specimens chosen for Figure $4 C-F$ were smooth-eyed. These findings show that TeTxLC has some influence on eye roughness in this genetic background. However, the optic lobe phenotypes described in this study are independent of eye roughness in GMRGal4/UAS-TNT-H flies.

\section{Neuronal synaptobrevin is necessary for neuropil pattern formation and CAM regulation as revealed by genetic mosaics}

The cleavage of n-syb is the only known function of TeTxLC (Sweeney et al., 1995), whereas even the n-syb homologous synaptobrevin isoform syb-a, which occurs in non-neuronal cells (Chin et al., 1993), remains uncleaved in vitro Thus, n-syb null tissues should exhibit the same phenotypes as tissue in which TeTxLC is expressed. To confirm this hypothesis, we investigated n-syb hypomorph pupae and n-syb null eye mosaics as well as the staining pattern of $n$-syb throughout morphogenesis.

We used an affinity-purified antiserum against the intravesicular tail of n-syb, an oligopeptide that shares no homology with the ubiquitously expressed synaptobrevin in Drosophila (Deitcher et al., 1998), to stain brains of wild-type larvae and pupae at $\mathrm{P}+15$, $\mathrm{P}+25, \mathrm{P}+50$, and $\mathrm{P}+90 \%$ as well as adults. In L3 larvae, the larval central brain, the larval optic neuropil, and ventral ganglion exhibit high immunoreactivity. The developing optic lobes are only faintly recognizable, revealing an obvious difference between active and inactive neuropils (Fig. $5 A$ ). At $\mathrm{P}+15 \%$ (Fig. $5 B)$ and $\mathrm{P}+25 \%$ neuropil structures of the optic lobe are already weakly stained. All neuropil structures exhibit high n-syb immunoreactivity from $\mathrm{P}+50 \%$ onward, at the end of pupation (Fig. $5 C$ ) and during adulthood. The early increase of n-syb after $\mathrm{P}+$ $25 \%$ coincides with the time window of photoreceptor terminal pattern refinement.

To test the influence of lowered n-syb levels during development, we analyzed n-syb hypomorph mutants (Deitcher et al., 1998). Stainings of n-syb hypomorph adults with anti-chaoptin reveal no significant anomalies of photoreceptor morphology, and stainings of IrreC-rst show normally downregulated immunore- activity (data not shown). Anti-FasII stainings of hypomorph adults reveal slightly increased Fasciclin II immunoreactivity in lamina monopolar cells L1 and L3 (data not shown). In wild type these cells exhibit high Fasciclin II immunoreactivity only during metamorphosis. However, the quantity of n-syb in these hypomorph mutants is unknown, as are putative thresholds for the occurrence of either the morphological or the CAM regulation phenotypes. Flies with a complete lack of n-syb in optic tissues are therefore desirable.

Because the homozygous n-syb null allele is embryonic lethal (Deitcher et al., 1998), we produced n-syb null eye mosaics to study photoreceptor neurons without n-syb expression. Mosaic clones were created in first instar eye imaginal disks by x-rayinduced somatic recombination. We screened $\sim 5000$ adult flies, of which 50 exhibited eye mosaics. Most of these had twin spots (red and white patches) within the otherwise orange eyes of the heterozygous genetic background. However, some flies exhibited only white spots, and others exhibited only red ones. Only individuals with twin spots or white eye spots were photographed and subsequently analyzed by staining with the antibodies against Fasciclin II, IrreC-rst, and chaoptin.

Anti-FasII staining reveals high Fasciclin II immunoreactivity of $\mathrm{R} 7$ and $\mathrm{R} 8$ projections in the distal medulla (Fig. $6 \mathrm{C}$ ) in an area corresponding to the white eye spot and a lamina neuropil patch. The morphology of these photoreceptor arborizations is significantly disturbed. The strong Fasciclin II immunoreactivity in n-syb null photoreceptors is coherent with the increased levels of Fasciclin II on photoreceptors expressing TeTxLC (Fig. 4D).

Anti-IrreC staining reveals no alteration of the IrreC-rst expression pattern. This finding corresponds to the anti-IrreC stainings of n-syb hypomorphs as well as to adults expressing toxin in photoreceptors, in which no altered IrreC-rst levels are found (data not shown).

Stainings with anti-Chaoptin reveal a significant difference between n-syb null photoreceptors and surrounding photoreceptors. Corresponding to the position and shape of a white eye spot, significantly stronger staining of $\mathrm{R} 7$ and $\mathrm{R} 8$ projections in the medulla can be detected (Fig. $6 A, B$ ). This shows a dependence of chaoptin regulation on functional n-syb. After Fasciclin II, cha- 


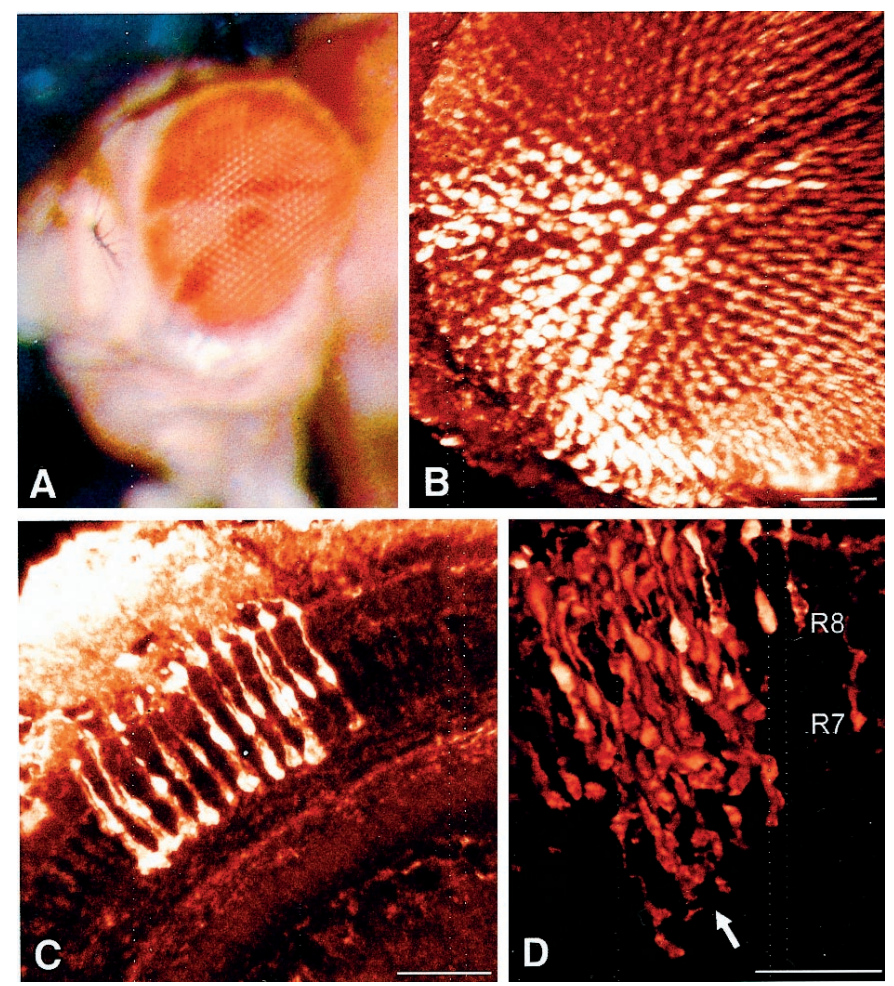

Figure 6. n-syb null eye mosaics. A, Twin spot in a fly's eye. The white patch marks photoreceptors without neuronal synaptobrevin; red patches correspond to homozygous wild-type synaptobrevin; and orange marks the heterozygous background. $B$, View onto a three-dimensional reconstruction of the distal medulla (viewed from the eye) of the same specimen as in $A$ in a staining with anti-chaoptin. Although all photoreceptor terminals exhibit chaoptin immunoreactivity, a patch of exactly the same shape as the white eye spot exhibits significantly stronger immunoreactivity. $C$, Anti-FasII staining of another specimen with a white eye spot. A restricted area of strong Fasciclin II immunoreactivity in photoreceptors is visible. $D, 3 \mathrm{D}$ reconstruction of a patch of Fasciclin II-positive photoreceptor terminals in the distal medulla reveals many overlaps of arborizations (arrow). R7, R8, Photoreceptor terminals. Scale bars, $20 \mu \mathrm{m}$.

optin thus is the second CAM with a regulation that depends on $\mathrm{n}$-syb expression in photoreceptors.

\section{DISCUSSION}

We studied the effects of interfering with synaptic machinery during pupal optic lobe development in Drosophila. Neuronal synaptobrevin was chosen as a target of interference because of (1) the availability of genetic tools and information for cellspecific "knock-out" and (2) its crucial role for the function of synapses. We showed the influence of a lack of functional n-syb on the developing optic lobe by targeted tetanus toxin light chain expression and by generating n-syb null eye mosaics. Although cell survival, axonal pathfinding, and target recognition are unaffected by the absence of functional n-syb, morphological alterations of photoreceptor terminals between $\mathrm{P}+25$ and $\mathrm{P}+50 \%$ as well as the dependence of Fasciclin II and chaoptin regulation in photoreceptors on neuronal synaptobrevin are demonstrated.

\section{Specificity and cell-autonomous function of tetanus toxin light chain}

We showed in this study that widespread expression of TeTxLC in the optic lobe has no influence on axonal pathfinding and cell survival. The morphological and CAM regulation phenotypes observed in TeTxLC-expressing photoreceptors could be confirmed to result from a lack of neuronal synaptobrevin.

Another study of TeTxLC expression in photoreceptors revealed movement blindness in a behavioral paradigm, which can well be explained with nonfunctional n-syb (A. Keller and M. Heisenberg, personal communication). Expression of TeTxLC in a characterized neuronal circuit of the CNS also produces a specific behavioral defect (Reddy et al., 1997).

The observation that L1 and L3 neurons form normal terminals and express Fasciclin II at wild-type levels when TeTxLC is expressed in photoreceptors, their major synaptic input, implies that both the morphological and the CAM regulation phenotypes develop independently of neuronal input. Our results suggest that only those neurons are affected in which n-syb function is disabled, but not cells that are immediately postsynaptic.

In flies that express active toxin in photoreceptors an increased occurrence of rough eyes can be observed. The facts that (1) already the parental GMR-Gal4 flies but not UAS-TNT flies have a severe eye phenotype, (2) eye roughness of the F1 flies does not occur with high penetrance, and (3) all phenotypes presented in this paper could be shown to be independent of eye roughness together indicate that the influence of TeTxLC on eye patterning may be an enhancement of an already present defect in GMRGal4. Because n-syb null eye mosaics do not exhibit any differences in eye patterning between white patches and the rest of the eye, n-syb is not required for this process.

\section{Role of neuronal synaptobrevin during optic lobe development}

The finding of an onset of n-syb expression in the first half of pupation poses the question of whether synapses actually start to function so early during optic lobe development. Neuronal activity plays a major role during vertebrate visual system development (for review, see Shatz, 1996). A critical period of $1 \mathrm{~d}$ after eclosion for experience-dependent developmental plasticity in the Drosophila lamina was demonstrated by Barth et al. (1997). It has not yet been shown whether synaptic plasticity in the Drosophila CNS extends to pupation or whether neurotransmitters are released before any form of neuronal activity. Assuming the involvement of such processes, we would expect the following time scale: first, expression and localization of proteins of the vesicle release machinery; second, release of neurotransmitter independent or dependent on spontaneous activity; and third, release of neurotransmitter dependent on evoked activity. Given the early immunoreactivity of specific synaptic vesicle cycle proteins such as $\mathrm{n}$-syb and synaptotagmin before $\mathrm{P}+25 \%$, the synaptic vesicle cycle appears to be available for more than half of pupal development before first evoked photoreceptor responses occur at $\mathrm{P}+$ $82 \%$. Morphological analysis revealed a brief interval of intense synapse formation in the lamina of Musca starting $\sim \mathrm{P}+62 \%$ and peaking at $\mathrm{P}+74 \%$ (Fröhlich and Meinertzhagen, 1983). Although this time window does not necessarily correspond to the first occurrence of synapses in the optic lobe of Drosophila, and the heterogeneity of optic lobe neurons should be considered, it may indicate that $n$-syb is expressed long before synapses are morphologically recognizable.

Apparently, not all processes between target selection and the establishment of functional connectivity are yet known. The demonstration of the dependence of neuropil patterning on NO release (Gibbs and Truman, 1998) shows a process of terminal development in a similar time window as the neuropil patterning 
defects we observed. With regard to the current study, one possibility would be the involvement of n-syb in the release of neurotransmitters or other factors before or during synapse development. Li et al. (1997) observed that histamine is synthesized in photoreceptors extending from cultured imaginal disks. Histamine or other substances released by growth cones after arrival in their target layers might exert functions necessary for the establishment of a regular terminal pattern.

Lack of functional n-syb has no obvious influence on target selection and the development of largely overlapping terminals of R7 and R8 cells. In contrast, further development of terminal fine structure between $\mathrm{P}+25$ and $\mathrm{P}+50 \%$ is significantly disturbed, indicating its involvement in a fine-tuning process. This early onset of $n$-syb function shows that either $n$-syb is involved in nonsynaptic processes taking place soon after target recognition, or synapses form earlier in the Drosophila optic lobe than is generally believed. Because this time window lies significantly before the observed upregulation of Fasciclin II in active toxinexpressing photoreceptors, the morphological changes do not depend on this CAM.

\section{Role of cell adhesion molecules during optic lobe development}

Cell adhesion molecules play multiple roles during optic lobe development. Best investigated are functions during axon guidance and target recognition (for review, see Tessier-Lavigne and Goodman, 1996) and synaptic plasticity (for review, see Martin and Kandel, 1996).

Our finding of increased Fasciclin II immunoreactivity under conditions of blocked neurotransmitter release corresponds to earlier studies that showed the opposite effect with an opposite approach: apCAM is downregulated after application of serotonin (Mayford et al., 1992), and synaptic Fasciclin II is reduced in mutants with abnormally high neuronal activity (Schuster et al., 1996b). Although it was demonstrated for apCAM that it is downregulated via endocytosis (Bailey et al., 1992), the mechanism of activity-dependent Fasciclin II downregulation at the Drosophila neuromuscular junction remains unknown. Possible downregulation mechanisms to be considered include endocytosis, extracellular cleavage, and reduced transcription or translation in combination with a continuous turnover of the protein.

The upregulation of two different types of CAMs (Fasciclin II and chaoptin) in the same cell type under conditions of blocked neurotransmitter release poses the question of the specificity of the mechanism. In the absence of functional n-syb, increased numbers of docked synaptic vesicles accumulate presynaptically (Broadie et al., 1995). Assuming that this would result in a significant sequestration of membrane material and that the synaptic vesicle cycle was continuously replenished from cell surfaces carrying adhesion molecules, deactivation of n-syb could result in decreased intake of CAMs and thus increased CAM immunoreactivity. However, current understanding of the recycling mechanism in the synaptic vesicle cycle (Südhoff, 1995) and different localization of CAM isoforms (for review, see Martin and Kandel, 1996) does not support this hypothesis. Alternatively, specifically CAMs on active terminals and fibers could be downregulated to serve as markers for the competence of the synapses for sprouting (as suggested by Schuster et al., 1996b).

In wild-type third instar larvae Fasciclin II is found on R7 and R8 retinal axons (Kaphingst and Kunes, 1994). During parts of pupation, Fasciclin II is detectable at low levels on photoreceptor cell bodies (Reiter, unpublished results). We think it possible that
Fasciclin II is never completely downregulated from R7 and R8 terminals but is mostly below threshold for visualization with confocal microscopy. Upregulation of Fasciclin II levels in photoreceptors lacking functional $\mathrm{n}$-syb after $\mathrm{P}+75 \%$ may thus be attributable to an accumulation of the protein, when its downregulation would normally occur via an n-syb-dependent mechanism as part of a continuous protein turnover.

The finding that IrreC-rst immunoreactivity remains unaltered in photoreceptors without functional n-syb but is increased in proximal neuropils after widespread TeTxLC expression can be interpreted in two different ways: either IrreC-rst protein is not present on photoreceptor terminals at the addressed time of pupation, or the n-syb-dependent CAM downregulation mechanism has a different molecular specificity in photoreceptors than in other optic lobe cells. During axonal pathfinding IrreC-rst is expressed on photoreceptors (Schneider et al., 1995; Reiter et al., 1996). In pupal stages IrreC-rst is shown to be localized on rhabdomeres but not on axons and cell bodies of photoreceptors during the second half of pupation. Because rhabdomeres are unique to this cell type and seem to be a preferred localization for IrreC-rst in photoreceptors, a cell-specific distribution that excludes terminals appears more likely than a specific CAM regulation mechanism for photoreceptors.

Taken together, our results clearly show a requirement of n-syb for optic lobe development. Either n-syb has a previously unknown activity-independent function, or synaptic transmission is involved in optic lobe development, or both. Investigations of the underlying regulatory processes, immunoelectron microscopic analysis of CAM localization at neuron-neuron synapses and a possible causal link to neuronal activity during CNS development now have to be undertaken.

\section{REFERENCES}

Ashley JA, Katz FN (1994) Competition and position-dependent targeting in the development of the Drosophila R7 visual projections. Development 120:1537-1547.

Bailey CH, Chen M, Keller F, Kandel ER (1992) Serotonin-mediated endocytosis of apCAM: an early step of learning-related synaptic growth in Aplysia. Science 256:645-649.

Barth M, Hirsch HVB, Meinertzhagen IA, Heisenberg M (1997) Experience-dependent developmental plasticity in the optic lobe of Drosophila melanogaster. J Neurosci 17:1493-1505.

Brand AH, Dormand E-L (1995) The Gal4 system as a tool for unravelling the mysteries of the Drosophila nervous system. Curr Opin Neurobiol 5:572-578.

Broadie K, Prokopp A, Bellen HJ, O'Kane CJ, Schulze KL, Sweeney ST (1995) Syntaxin and synaptobrevin function downstream of vesicle docking in Drosophila. Neuron 15:663-673.

Broadie K, Bate M (1993) Activity-dependence of the neuromuscular synapse during Drosophila embryogenesis. Neuron 11:607-619.

Chin AC, Burgess RW, Wong BR, Schwarz TL, Scheller RH (1993) Differential expression of transcripts from syb, a Drosophila melanogaster gene encoding VAMP (synaptobrevin) that is abundant in nonneuronal cells. Gene 131:175-181.

Davis GW, Schuster CM, Goodman CS (1997) Genetic analysis of the mechanisms controlling target selection: target-derived Fasciclin II regulates the pattern of synapse formation. Neuron 19:561-573.

Deitcher DL, Ueda A, Stewart BA, Burgess RW, Kidokoro Y, Schwarz TL (1998) Distinct requirements for evoked and spontaneous release of neurotransmitter are revealed by mutations in the Drosophila gene neuronal-synaptobrevin. J Neurosci 18:2028-2039.

DiAntonio A, Burgess RW, Chin AC, Deitcher DL, Scheller RH, Schwarz TL (1993) Identification and characterization of Drosophila genes for synaptic vesicle proteins. J Neurosci 13:4924-4935.

Fischbach K-F, Technau G (1984) Cell degeneration in the developing optic lobes of the sine oculis and small-optic-lobes mutants of Drosophila melanogaster. Dev Biol 104:219-239. 
Freeman M (1996) Reiterative use of the EGF receptor triggers differentiation of all cell types in the Drosophila eye. Cell 87:651-660.

Fröhlich A, Meinertzhagen IA (1982) Synaptogenesis in the first optic neuropil of the fly's visual system. J Neurocytol 11:159-180.

Fröhlich A, Meinertzhagen IA (1983) Quantitative features of synapse formation in the fly's visual system. J Neurosci 11:2336-2349.

Gavrieli Y, Sherman Y, Ben-Sasson SA (1992) Identification of programmed cell death in situ via specific labeling of nuclear DNA fragmentation. J Cell Biol 119:493-501.

Gibbs SM, Truman JW (1998) Nitric oxide and cyclic GMP regulate retinal patterning in the optic lobe of Drosophila. Neuron 20:83-93.

Goodman CS, Shatz C (1993) Developmental mechanisms that generate precise patterns of neuronal connectivity. Cell 72:77-98.

Grenningloh G, Rehm EJ, Goodman CS (1991) Genetic analysis of growth cone guidance in Drosophila: Fasciclin II functions as a neuronal recognition molecule. Cell 67:45-57.

Hardie RC, Peretz A, Pollock JA, Minke B (1993) Ca2+ limits the development of the light response in Drosophila photoreceptors. Proc R Soc Lond B Biol Sci 252:223-229.

Kaphingst K, Kunes S (1994) Pattern formation in the visual centers of the Drosophila brain: wingless acts via decapentaplegic to specify the dorsoventral axis. Cell 78:437-448.

Krantz DE, Zipursky SL (1990) Drosophila chaoptin, a member of the leucine-rich repeat family, is a photoreceptor cell-specific adhesion molecule. EMBO J 9:1969-1977.

Laissue PP, Reiter C, Hiesinger PR, Halter S, Fischbach K-F, Stocker RF (1999) Three-dimensional reconstruction of the antennal lobe in Drosophila melanogaster. J Comp Neurol 405:543-552.

Li C, Meinertzhagen IA (1997) The effects of 20-hydroxyecdysone on the differentiation in vitro of cells from the eye imaginal disc from Drosophila melanogaster. Invert Neurosci 3:57-69.

Lin DM, Goodman CS (1994) Ectopic and increased expression of Fasciclin II alters motoneuron growth cone guidance. Neuron 13:507-523.

Martin KC, Kandel ER (1996) Cell adhesion molecules, CREB, and the formation of new synaptic connections. Neuron 17:567-570.

Mayford M, Barzilai A, Keller F, Schacher S, Kandel ER (1992) Modulation of an NCAM-related adhesion molecule with long-term synaptic plasticity in Aplysia. Science 256:638-644.

Meinertzhagen IA, Hanson TE (1993) The development of the optic lobe. In: The development of Drosophila melanogaster, Vol II, pp 1363-1491. Cold Spring Harbor, NY: Cold Spring Harbor Laboratory. Meinertzhagen IA, O'Neil SD (1991) Synaptic organization of columnar elements in the lamina of the wild type in Drosophila melanogaster. J Comp Neurol 305:232-263.

Moses K, Rubin GM (1991) Glass encodes a site-specific DNA-binding protein that is regulated in response to positional signals in the developing Drosophila eye. Genes Dev 5:583-593.

Reddy S, Jin P, Trimarchi J, Caruccio P, Phillis R, Murphey RK (1997) Mutant molecular motors disrupt neural circuits in Drosophila. J Neurobiol 33:711-723.

Reiter C, Schimansky T, Nie Z, Fischbach K-F (1996) Reorganization of membrane contacts prior to apoptosis in the Drosophila retina: the role of the IrreC-rst protein. Development 122:1931-1940.

Schneider T, Reiter C, Eule E, Bader B, Lichte B, Nie Z, Schimansky T, Ramos GP, Fischbach K-F (1995) Restricted expression of the IrreCrst protein is required for normal axon projections of columnar visual neurons. Neuron 15:259-271.

Schuster CM, Davis GW, Fetter RD, Goodman CS (1996a) Genetic dissection of structural and functional components of synaptic plasticity. I. Fasciclin II controls synaptic stabilization and growth. Neuron 17:641-654.

Schuster CM, Davis GW, Fetter RD, Goodman CS (1996b) Genetic dissection of structural and functional components of synaptic plasticity. II. Fasciclin II controls presynaptic structural plasticity. Neuron 17:655-667.

Shatz CJ (1996) Emergence of order in visual system development. Proc Natl Acad Sci USA 93:602-608.

Südhoff TC (1995) The synaptic vesicle cycle: a cascade of proteinprotein interactions. Nature 375:645-653.

Sweeney ST, Broadie K, Keane J, Niemann H, O'Kane CJ (1995) Targeted expression of tetanus toxin light chain in Drosophila specifically eliminates synaptic transmission and causes behavioral defects. Neuron 14:341-351.

Tessier-Lavigne M, Goodman CS (1996) The molecular biology of axon guidance. Science 274:1123-1133.

Van Vactor Jr D, Krantz DE, Reinke R, Zipursky SL (1988) Analysis of mutants in chaoptin, a photoreceptor cell-specific glycoprotein in Drosophila, reveals its role in cellular morphogenesis. Cell 52:281-290.

Wu MN, Bellen HJ (1997) Genetic dissection of synaptic transmission in Drosophila. Curr Opin Neurobiol 7:624-630.

Zipursky SL, Venkatesh TR, Teplow DB, Benzer S (1984) Neuronal development in the Drosophila retina: monoclonal antibodies as molecular probes. Cell 36:15-26. 